\title{
Effects of the pseudo-Chern-Simons action for strongly correlated electrons in a plane
}

\author{
R. F. Ozela $\odot,{ }^{*}$ Van Sérgio Alves $\odot,{ }^{\dagger}$ G. C. Magalhães, ${ }^{\ddagger}$ and Leandro O. Nascimento ${ }^{\S}$ \\ Faculdade de Física, Universidade Federal do Pará, 66075-110 Belém, PA, Brazil
}

(Received 28 October 2021; accepted 4 February 2022; published 7 March 2022)

\begin{abstract}
Chiral symmetry breaking comes from the mass dynamically generated through interaction of Dirac fermions for both quantum electrodynamics in $(2+1) \mathrm{D}(\mathrm{QED} 3)$ and $(3+1) \mathrm{D}(\mathrm{QED} 4)$. In QED3, the presence of a Chern-Simons (CS) parameter affects the critical structure of the theory, favoring the symmetric phase where the electron remains massless. Here, we calculate the main effects of a pseudoChern-Simons (PCS) parameter $\theta$ into the dynamical mass generation of pseudo-quantum electrodynamics (PQED). The $\theta$ parameter provides a mass scale for PQED at the classical level and appears as the pole of the gauge-field propagator. After calculating the full electron propagator with the Schwinger-Dyson equation in the quenched-rainbow and large- $N$ approximations, we conclude that $\theta$ changes the critical parameters related to the fine-structure constant $\alpha_{c}(\theta)$ and the number of copies of the matter field $N_{c}(\theta)$, favoring the symmetric phase. In the continuum limit $(\Lambda \rightarrow \infty)$, nevertheless, the $\theta$ parameter does not affect the critical parameters. We also compare our analytical results with numerical findings of the integral equation for the mass function of the electron. In the strong-coupling limit (with a fine-structure constant $\alpha \gg 1$ ), the PCS mass vanishes and the system presents the same criticality as QED3.
\end{abstract}

DOI: $10.1103 /$ PhysRevD.105.056004

\section{INTRODUCTION}

In the last few decades, quantum field theories in $(2+1) \mathrm{D}$ have been extensively studied. This interest is partly due to its potential applications in condensed matter physics [1-24] and, for comparison with quantum chromodynamics, at low-energy scales. In particular, QED3 has provided interesting features that are similar to quantum chromodynamics, such as dynamical mass generation [25-36] and confinement [37-39].

Pseudo quantum electrodynamics (PQED) is the dimensional reduction of QED4, when the matter field is constrained to move within a plane and the photons are allowed to propagate away from and back to this plane; as such, it is a unitary [40] model that respects causality [41,42] and describes mixed-dimensionality systems. Because of that, $2 \mathrm{~d}$ materials - whose electromagnetic interactions escape the plane, as happens in graphene [43-48], silicene [49], and transition metal dichalcogenides [49-53]—are perfect

\footnotetext{
*ozelarf@gmail.com

†ansergi@ufpa.br

gabrielconduru@hotmail.com

§lon@ufpa.br
}

Published by the American Physical Society under the terms of the Creative Commons Attribution 4.0 International license. Further distribution of this work must maintain attribution to the author(s) and the published article's title, journal citation, and DOI. Funded by SCOAP . candidates to be described by the mixed-dimensionality electromagnetic description of pseudo-quantum electrodynamics (PQED) and end up defining a relevant breakthrough to this research topic. Within the myriad of results PQED has given rise to, we allude in hindsight to dynamical mass generation for fermions at zero and finite temperature [54-56], the interaction-driven quantum valley Hall effect [57], quantum corrections of the electron $g$-factor [58], electron-hole pairing (excitons) in transition metal dichalcogenides $[59,60]$, the optical infrared conductivity of graphene [61], the emergence of a dynamically generated mass for PQED with the Gross-Neveu interaction [62], Yukawa potential in the plane [63,64], and PQED cavity effects $[65,66]$.

For massless Dirac particles, dynamical mass generation has been investigated in several scenarios in both QED4 [67-81] and QED3 [25-36], providing a critical value either for the fine-structure constant or for the number of flavors, respectively. This nonperturbative effect is usually calculated with the Schwinger-Dyson (SD) equations for the full electron propagator [82-84]. For graphene, this dynamical mass generation implies a gap opening at the Dirac points of the quasiparticle excitation [57], which might cause a topological phase transition [22].

Meanwhile, the Chern-Simons (CS) term plays a vital role in gauge field topological phase transitions. Indeed, it generates a mass for the gauge field while breaking the parity symmetry and preserving the gauge symmetry [85]. 
As a consequence, the CS term's applications to the Meissner effect are documented in the literature [86-91].

The addition of the CS term to QED3 has been shown to favor the symmetric phase, where the electron remains massless in both representations for spinors [92-94]. On the other hand, coupled to PQED, the CS term changes the electric permittivity of the vacuum-effectively working as a dielectric medium [95,96] — and it has been used to calculate the nonperturbative mass generation for the fermions [97]. This CS parameter, however, is dimensionless and cannot generate a mass for the gauge field $\mathrm{i}$ $n$ PQED. In order to do so, one has to consider the pseudoChern-Simons (PCS) action, obtained by dual transformation of an Abelian Chern-Simons-Higgs [98] or by a bosonization of massive Dirac fermions [99]. We shall refer to the model that combines PQED and PCS as pseudoMaxwell-Chern-Simons (PMCS) theory. This PMCS has a peculiar feature of producing bound states of electrons $[98,99]$. Nevertheless, effects of dynamical mass generation had not been investigated for PMCS theory until now.

In this work, we use the Schwinger-Dyson equations to investigate the dynamical mass generation associated with four-component fermions coupled to the PCS terms. We use the so-called quenched-rainbow approach and the large- $N$ expansion for calculating the mass function $\Sigma(p)$-i.e., the term of the electron self-energy that yields the dynamical mass. Our main results show that there exist a critical coupling constant $\alpha_{c}(\theta)$ and a critical number of fermions $N_{c}(\theta)$ separating the broken phase from the symmetric (massless) phase. It is shown that these are dependent on the PCS parameter $\theta$. Thereafter, we compare our approximated analytical results with the numerical results obtained from the integral equation for the mass function and illustrate some of its interesting properties. Because $\theta$ has the dimension of mass in the PMCS model, it essentially means an effective correlation length and, therefore, our results may be relevant for describing a symmetry restoration in two-dimensional materials due to a screening of the electromagnetic interaction.

This paper is organized as follows: In Sec. II, we present the model and set up the coupled SD equations for the photon and fermion propagators. In Sec. III, we present the truncation scheme using the quenched-rainbow approximation and investigate the analytical solutions of the integral equation for the mass function of fermions. In Sec. IV, we examine the influence of vacuum polarization on the CSB using the $N$ massless fermion flavors version and adopting a $1 / N$ expansion. In Sec. V, we plot the mass function for the electron numerically and characterize the different regimes found. We summarize our results in Sec. VI. In Appendix A, we review some aspects of the criticality of QED3 coupled to the usual CS term, and in Appendix B, we discuss some details of the analytical approximation performed.

\section{THE MODEL AND ITS TRUNCATED SCHWINGER-DYSON EQUATION}

We start with a PMCS model, where the CS term is modified by a pseudo-differential operator. This action has been shown to be dual to the Chern-Simons-Higgs model in Ref. [98] using standard path-integral formalism. We represent the PMCS model by

$$
\begin{aligned}
\mathcal{L}_{\mathrm{PMCS}}= & \frac{1}{2} \frac{F_{\mu \nu} F^{\mu \nu}}{\sqrt{-\square}}+\frac{i \theta}{2} \frac{\epsilon_{\mu \nu \gamma} A^{\mu} \partial^{\nu} A^{\gamma}}{\sqrt{-\square}} \\
& +\bar{\psi} i \not \partial \psi+\frac{\lambda}{2} \frac{\left(\partial_{\mu} A^{\mu}\right)^{2}}{\sqrt{-\square}}+e \bar{\psi} \gamma^{\mu} \psi A_{\mu},
\end{aligned}
$$

where $A^{\mu}$ is a gauge field, $\theta$ is the CS massive parameter, $e$ is the dimensionless coupling constant, $1 / \sqrt{-\square}$ is a pseudo-differential operator, and the fermions are represented by the four-component spinors $\psi$ and $\bar{\psi}$, describing the Dirac field. We expect to find nonzero values of $\theta$ in mixed-dimensionality systems that exhibit a superconducting phase, as discussed in Ref. [99] (where it is shown that $\theta$ is intrinsically associated with an attractive potential between the electrons and controls the maximum depth of the static potential).

This gives rise to a bare gauge-field propagator given by

$$
\Delta_{\mu \nu}^{(0)}(p)=\frac{p^{2} P_{\mu \nu}+\theta \epsilon_{\mu \nu \gamma} p^{\gamma}}{2 \varepsilon \sqrt{p^{2}}\left(p^{2}+\theta^{2}\right)}+\Delta_{\mu \nu}^{G F}(p),
$$

where $\varepsilon$ is included in order to describe the dieletric constant, and $\Delta_{\mu \nu}^{G F}(p)$ represents the gauge-dependent part which is null in the Landau gauge $\lambda \rightarrow \infty$. The fermion bare propagator is given by

$$
S_{F}^{(0)}(p)=-\frac{\not p}{p^{2}}
$$

and an interaction vertex by $\Gamma_{\mu}^{(0)}=e \gamma_{\mu}$.

The main consequence of the $\theta$ parameter is to provide a bounded pair of electrons [98] within a typical distance of $r_{0} \propto 1 / \theta$, as explained in Ref. [99]. Furthermore, such a parameter does not change the main results of the renormalization group functions at one-loop expansion, because it appears in any divergent term of the two-point vertex functions associated with Eq. (1). Next, we shall calculate the main contribution of $\theta$ through a nonperturbative approach, provided by the Schwinger-Dyson equation in the ladder approximation.

In order to investigate the corrections coming apace with the PMCS dynamics, we consider the following coupled Schwinger-Dyson equations displayed in Fig. 1 and analytically written as

$$
\left[S_{F}(p)\right]^{-1}=\left[S_{F}^{(0)}(p)\right]^{-1}-\Xi(p)
$$




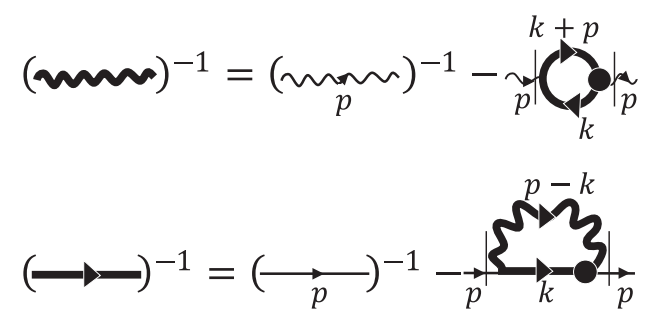

FIG. 1. The fermion and photon SD equations, respectively. The bold and filled dots indicate full propagators and vertices. The second terms on the right-hand side represent the photon selfenergy $\Pi^{\mu \nu}(p)$ (top) and the electron self-energy $\Xi(p)$ (bottom).

and

$$
\Delta_{\mu \nu}^{-1}(p)=\Delta_{0 \mu \nu}^{-1}(p)-\Pi_{\mu \nu}(p)
$$

where the electron and photon self-energies are, respectively,

$$
\Xi(p)=e^{2} \int \frac{d^{3} k}{(2 \pi)^{3}} \gamma^{\mu} S_{F}(k) \Gamma^{\nu}(k, p) \Delta_{\mu \nu}(p-k)
$$

and

$\Pi^{\mu \nu}(p)=-e^{2} \int \frac{d^{3} k}{(2 \pi)^{3}} \operatorname{Tr}\left[\gamma^{\mu} S_{F}(k+p) \Gamma^{\nu}(k, p) S_{F}(k)\right]$

In the Euclidean spacetime, we can write

$$
\left[S_{F}(p)\right]^{-1}=-\gamma^{\mu} p_{\mu} A(p)+\Sigma(p)
$$

where $A(p)$ is the wave function renormalization and $\Sigma(p)$ is the mass function. Posteriorly, substituting Eq. (8) into Eq. (4), we find that

$$
\begin{aligned}
& -\gamma^{\alpha} p_{\alpha} A(p)+\Sigma(p) \\
& =-\gamma^{\alpha} p_{\alpha}+-e^{2} \int \frac{d^{3} k}{(2 \pi)^{3}} \frac{\gamma^{\mu} S_{F}(k) \Gamma^{\nu}(k, p) \Delta_{\mu \nu}(p-k)}{\gamma^{\beta} p_{\beta} A(k)+\Sigma(k)}
\end{aligned}
$$

From Eq. (9), we can isolate an expression for $A(p)$ by multiplying $\not x$ on both sides and then calculating the trace over the Dirac matrices, hence

$$
A(p)=1-\frac{e^{2}}{4 p^{2}} \int \frac{d^{3} k}{(2 \pi)^{3}} \operatorname{Tr}\left[\gamma^{\beta} p_{\beta} F(p)\right]
$$

where for the sake of simplicity we define

$$
F(p)=\left[\frac{\gamma^{\mu} S_{F}(k) \Gamma^{\nu}(k, p) \Delta_{\mu \nu}(p-k)}{\gamma^{\beta} p_{\beta} A(k)+\Sigma(k)}\right] .
$$

For $\Sigma(p)$, we simply take the trace over the $\gamma^{\mu}$ matrices in Eq. (9) to get

$$
\Sigma(p)=-e^{2} \int \frac{d^{3} k}{(2 \pi)^{3}} \operatorname{Tr}[F(p)] .
$$

In the following sections, we shall consider a set of approximations in order to obtain an analytical result for the mass function. This will allow us to discuss the dynamical breaking of chiral symmetry, as well as to calculate the critical behavior of the model in terms of the $\theta$ parameter.

\section{QUENCHED-RAINBOW APPROXIMATION}

The ladder order is obtained by the so-called quenchedrainbow approximation, which has been previously applied to investigate the criticality of the PQED in the $4 \times 4$ representation [54-56]. It imposes that the full vertex $\Gamma^{\mu}$ and the full gauge-field propagator $\Delta_{\mu \nu}$ can be exchanged by their bare counterparts $\gamma^{\mu}$ and $\Delta_{\mu \nu}^{(0)}$. This approach decouples Eqs. (6) and (7). Also, note from Eq. (10) that we can conclude that $A(p) \approx 1+\mathcal{O}\left(e^{2}\right)$. Keeping this in mind, and using the trace properties for the Dirac matrices-namely, $\operatorname{Tr}(\mathbb{1})=4, \operatorname{Tr}\left(\gamma^{\mu} \gamma^{\nu}\right)=-4 \delta^{\mu \nu}$, and $\operatorname{Tr}\left(\gamma^{\mu} \gamma^{\nu} \gamma^{\beta}\right)=0$-we may calculate $\Sigma(p)$ from Eq. (12); hence,

$$
\Sigma(p)=e^{2} \int \frac{d^{3} k}{(2 \pi)^{3}} \Sigma(k) \delta^{\mu \nu} \Delta_{\mu \nu}^{(0)}(p-k) .
$$

Substituting Eq. (2) into Eq. (13) and using spherical coordinates, we obtain that, in the Landau gauge,

$$
\begin{aligned}
\Sigma(p)= & \frac{\alpha}{2 \pi^{2}} \int_{0}^{\Lambda} d k \frac{k^{2} \Sigma(k)}{k^{2}+\Sigma^{2}(k)} \int_{0}^{2 \pi} d \phi \\
& \times \int_{0}^{\pi} d \eta \frac{\sin (\eta) \sqrt{p^{2}+k^{2}-2 p k \cos (\eta)}}{p^{2}+k^{2}-2 p k \cos (\eta)+\theta^{2}},
\end{aligned}
$$

where $\Lambda$ is the UV cutoff, and $\alpha=e^{2} / 4 \pi \varepsilon$ is the finestructure constant. After solving the integrals over $\eta$ and $\phi$, we find

$$
\begin{aligned}
\Sigma(p)= & \frac{\alpha}{\pi} \int_{0}^{\Lambda} \frac{k \Sigma(k)}{k^{2}+\Sigma^{2}(k)}\left\{\frac{|p+k|-|p-k|}{p}\right. \\
& \left.+\frac{\theta}{p}\left[\arctan \left(\frac{|p-k|}{\theta}\right)-\arctan \left(\frac{|k+p|}{\theta}\right)\right]\right\} d k .
\end{aligned}
$$

We can split the integrand in Eq. (15) as a composition of kernels in the regions where $k \gg p$ and $k \ll p$, yielding 


$$
\begin{aligned}
\Sigma(p)= & \frac{\alpha}{\pi p} \int_{0}^{\Lambda} d k \frac{k \Sigma(k)}{k^{2}+\Sigma^{2}(k)}\left[\mathcal{K}_{k \gg p}(k, p) \Theta(k-p)\right. \\
& \left.+\mathcal{K}_{k \ll p}(k, p) \Theta(p-k)\right],
\end{aligned}
$$

where $\Theta(x)$ is the Heaviside function, while $\mathcal{K}_{k \gg p}(k, p)$ and $\mathcal{K}_{k \ll p}(k, p)$ are given by

$$
\mathcal{K}_{k \gg p}(k, p) \approx 2 p-\frac{2 p \theta^{2}}{k^{2}+\theta^{2}}
$$

and

$$
\mathcal{K}_{k \ll p}(k, p) \approx 2 k-\frac{2 k \theta^{2}}{p^{2}+\theta^{2}},
$$

respectively. As a consequence, the integrals are separated into

$$
\begin{aligned}
\Sigma(p)= & \frac{\alpha}{\pi p} \int_{0}^{p} \frac{2 k^{2} \Sigma(k)}{k^{2}+\Sigma^{2}(k)}\left[1-\frac{\theta^{2}}{k^{2}+\theta^{2}}\right] d k \\
& +\frac{\alpha}{\pi p} \int_{p}^{\Lambda} \frac{2 k p \Sigma(k)}{k^{2}+\Sigma^{2}(k)}\left[1-\frac{\theta^{2}}{k^{2}+\theta^{2}}\right] d k .
\end{aligned}
$$

After taking the derivative of Eq. (19) with respect to $p$ (see Appendix A for more details) and using the Leibniz integral rule [54-56], it becomes a nonlinear differential equation given by

$$
p^{2} \Sigma^{\prime \prime}(p)+2 p \Sigma^{\prime}(p)+\frac{2 \alpha}{\pi} \frac{p^{2}-\frac{p^{2} \theta^{2}}{p^{2}+\theta^{2}}}{p^{2}+\Sigma^{2}(p)} \Sigma(p)=0 .
$$

This is supplemented by two boundary conditionsnamely,

$$
\lim _{p \rightarrow \Lambda}\left[p \frac{d \Sigma(p)}{d p}+\Sigma(p)\right]=0
$$

for the UV regime and

$$
\lim _{p \rightarrow 0} p^{2} \frac{d \Sigma(p)}{d p}=0
$$

for the infrared regime.

Equation (20) has a nonlinear behavior in $p$, which prevents us from calculating an analytical solution for the mass function. This nonlinear feature is generated by the last nonzero term of Eq. (20). We could find linear differential equations by assuming either $p \ll \Sigma(p)$, but it is shown that the corresponding solution does not obey the boundary conditions, and is therefore an unphysical solution. Therefore, we will concentrate on the case of $p \gg \Sigma(p)$ and assume that $p \approx \Lambda$ (as discussed with more detail in Appendix B and in Refs. [54,100,101]) to find the linearized form $p^{2} \Sigma^{\prime \prime}(p)+2 p \Sigma^{\prime}(p)+\frac{2 \alpha}{\pi}\left[1-\frac{\theta^{2}}{\Lambda^{2}+\theta^{2}}\right] \Sigma(p)=0$,

a Euler differential equation solved by

$$
\Sigma(p)=A p^{\lambda_{+}}+B p^{\lambda_{-}}
$$

with

$$
\lambda_{ \pm}=\frac{-1 \pm \sqrt{1-\frac{\alpha}{\alpha_{c}}}}{2},
$$

where the critical value for the fine-structure constant has been defined as

$$
\alpha_{c}(\theta)=\frac{\pi \varepsilon}{\left(8-\frac{8 \theta^{2}}{\Lambda^{2}+\theta^{2}}\right)}=\underbrace{\alpha_{c}^{\mathrm{PQED}}}_{\pi \varepsilon / 8}\left(1+\frac{\theta^{2}}{\Lambda^{2}}\right) .
$$

Notice that for $\alpha<\alpha_{c}$, the solution in Eq. (24) does not satisfy the UV boundary condition and permits only a trivial solution that allows no chiral symmetry breaking, as deduced analogously for PQED in Ref. [54], where the value $\alpha_{c}^{\mathrm{PQED}}=\pi \varepsilon / 8$ has been deduced. For $\alpha>\alpha_{c}$, there is also a nontrivial solution similar to PQED that implies the emergence of a chiral symmetry breaking (see Appendix B). Turning $\theta$ off, we can hence see that Eq. (26) generalizes the result in the literature for PQED and indicates that the PCS mass $\theta$ might effectively be used to tune the critical point, as seen in Fig. 2.

The fact that $\Lambda$ also appears in Eq. (26) is a consequence of the scale invariance of the model when $\theta$ vanishes. In particular, when we take the continuum limit $(\Lambda \rightarrow \infty)$,

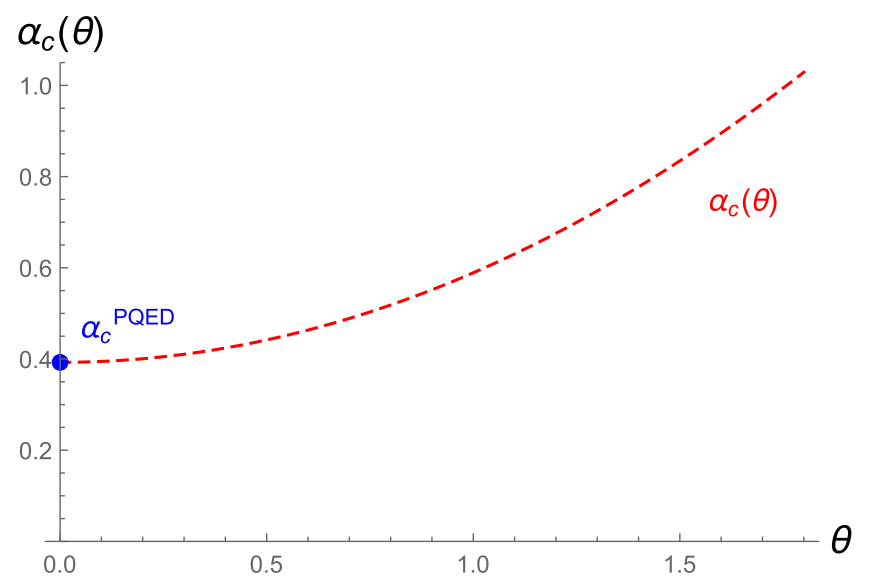

FIG. 2. The critical fine-structure constant as a function of $\theta$. We plot Eq. (26) with $\Lambda=10$ (units of energy) and $\varepsilon=1$. The blue dot is the critical fine-structure constant of PQED at $\theta=0$ (units of energy), which has been discussed in Refs. [54-56]. The dashed red line shows that as we increase $\theta$, the critical constant also increases, eventually bringing the system into its symmetric phase, where the mass function vanishes. 
the role of $\theta$ also disappears, as expected. Indeed, this represents a continuum limit, where the dynamical mass generation occurs for any value of $\alpha$, and therefore, we do not have an actual phase transition [54-56].

In this section, we have neglected the quantum corrections to the gauge-field propagator. Nevertheless, we may go beyond this by introducing the large- $N$ expansion, which is sometimes called the unquenched-rainbow approximation. Next, we consider this case.

\section{UNQUENCHED PMCS}

PQED has been shown to have a critical number of fermions $N_{c}$ which separates the symmetric from the broken phase [54-56], entailed when the number of copies of the fermionic field $N$ is greater than $N_{c}$. In this section, we investigate the role of the CS term in the criticality of PQED in the large- $N$ regime. Within the unquenchedrainbow approximation, we must extend the fermionic sector in Lagrangian (1) to $N$ copies of a massless fermion field; thus, we consider

$$
\begin{aligned}
\mathcal{L}_{\mathrm{PMCS}}= & \frac{1}{2} \frac{F_{\mu \nu} F^{\mu \nu}}{\sqrt{-\square}}+\frac{i \theta}{2} \frac{\epsilon_{\mu \nu \gamma} A^{\mu} \partial^{\nu} A^{\gamma}}{\sqrt{-\square}} \\
& +\sum_{a=1}^{N} \bar{\psi}^{a}\left(i \not \partial+e \gamma^{\mu} A_{\mu}\right) \psi^{a}+\frac{\lambda}{2} \frac{\left(\partial_{\mu} A^{\mu}\right)^{2}}{\sqrt{-\square}} .
\end{aligned}
$$

Applying a similar approach, it is possible to generalize such results to the PMCS model by adding the dominant order of the $1 / N$ expansion (which is equivalent to the random phase approximation; see Fig. 3) to the truncated gauge-field propagator in Sec. III. The gauge-field propagator for the PMCS model would then be

$$
\Delta_{\mu \nu}=T P_{\mu \nu}+L \epsilon_{\mu \nu \rho} p^{\rho}
$$

with

$$
\begin{aligned}
T= & \frac{8\left(p^{2}\right)^{3 / 2}+\left(8 \sqrt{p^{2}}-4 \Pi_{1}\right) \theta^{2}}{\left[4\left(p^{2}-\theta^{2}\right)+\Pi_{1}^{2}\right]^{2}+64 p^{2} \theta^{2}} \\
& +\frac{\Pi_{1}\left[5 p^{2}-\left(\Pi_{1}+\sqrt{p^{2}}\right)^{2}\right]}{\left[4\left(p^{2}-\theta^{2}\right)+\Pi_{1}^{2}\right]^{2}+64 p^{2} \theta^{2}}, \\
L= & \frac{2 \theta}{4\left[\left(p^{2}\right)^{3 / 2}-p^{2} \Pi_{1}+\theta^{2} \sqrt{p^{2}}\right]+\sqrt{p^{2}} \Pi_{1}^{2}},
\end{aligned}
$$

and

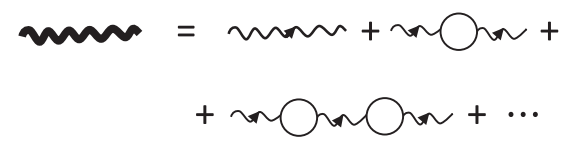

FIG. 3. Dominant order of the $1 / N$ expansion of a photon propagator.

$$
\Pi_{1}=-\frac{e^{2}}{8} N \sqrt{p^{2}}
$$

for massless fermions, as calculated in Refs. [102,103].

The gauge-field propagator in Eq. (28) simplifies to the PMCS propagator in Ref. [99]—namely,

$$
\Delta_{\mu \nu}(p)=\frac{p^{2} P^{\mu \nu}+\frac{16}{16+N e^{2}} \theta \epsilon_{\mu \nu \rho} p^{\rho}}{2 \varepsilon\left(1+\frac{N e^{2}}{16}\right) \sqrt{p^{2}}\left[p^{2}+\frac{\theta^{2}}{\left(1+\frac{N e^{2}}{16}\right)^{2}}\right.} .
$$

In order to compatibilize our model with the $1 / N$ expansion, we apply the transformation $e \rightarrow e / \sqrt{N}$ to get

$$
\Delta_{\mu \nu}=\frac{p^{2} P_{\mu \nu}+\tilde{\theta} \epsilon_{\mu \nu \rho} p^{\rho}}{2 \varepsilon_{e f} \sqrt{p^{2}}\left(p^{2}+\tilde{\theta}^{2}\right)},
$$

whereas the effective dielectric constant and effective CS parameter are, respectively,

$$
\begin{aligned}
\varepsilon_{e f} & =\varepsilon\left(1+\frac{e^{2}}{16}\right), \\
\tilde{\theta} & =\frac{\theta}{1+\frac{e^{2}}{16}} .
\end{aligned}
$$

Thus, similarly to Eq. (12), the mass function in the unquenched regime reads

$$
\Sigma(p)=\frac{e^{2}}{N} \int \frac{d^{3} k}{(2 \pi)^{3}} \frac{\Sigma(k) \delta^{\mu \nu} \Delta_{\mu \nu}(p-k)}{k^{2}+\Sigma^{2}(k)},
$$

where we use $A(p)=1$ because $A(p) \approx 1+\mathcal{O}(1 / N)$, and we are calculating $\Sigma(p)$ only up to the dominant order of the $1 / N$ expansion.

Since the propagator in Eq. (2) has the same overall structure as the PMCS propagator in Ref. [99], the only associations necessary to map the quenched approximation in Sec. III to an unquenched PMCS model are

$$
\begin{aligned}
\frac{2 \alpha}{\pi \varepsilon} & \rightarrow \frac{e^{2}}{2 \pi^{2} \varepsilon_{e f} N}, \\
\theta & \rightarrow \tilde{\theta} .
\end{aligned}
$$

Applied to Eq. (23), the transformations in Eq. (35) lead to $p^{2} \Sigma^{\prime \prime}(p)+2 p \Sigma^{\prime}(p)+\frac{e^{2}}{2 \pi^{2} \varepsilon_{e f} N}\left(1-\frac{\tilde{\theta}^{2}}{\Lambda^{2}+\tilde{\theta}^{2}}\right) \Sigma(p)=0$,

which satisfies the same boundary conditions as Eqs. (21) and (22). This allows us to identify a chiral-symmetrybroken phase (shaded region in Fig. 4) up to a critical number of fermions, 


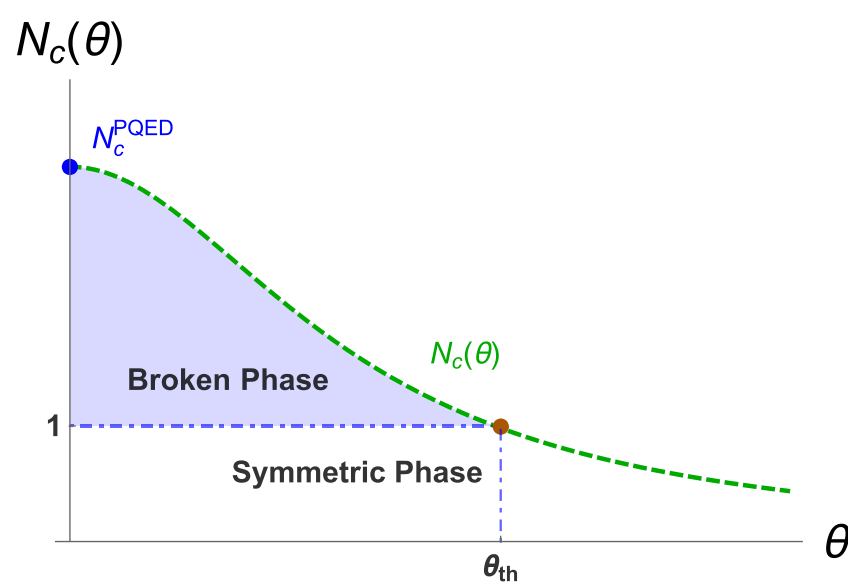

FIG. 4. Sketch of the maximum number of flavors that allow a manifestation of a broken phase as a function of $\theta$. The blue dot represents the critical number of flavors of PQED, where $\theta=0$ is deduced in Ref. [54-56] for $\varepsilon=1$. The dashed green line shows that as we increase $\theta$, the critical number of fermions decreases, guiding the system into its symmetric phase when $N_{c}$ gets smaller than 1.

$N_{c}(\theta)=\frac{2 e^{2}}{\pi^{2} \varepsilon_{e f}}\left(1-\frac{\tilde{\theta}^{2}}{\Lambda^{2}+\tilde{\theta}^{2}}\right)=N_{c}^{\mathrm{PQED}}\left(\frac{\Lambda^{2}}{\Lambda^{2}+\tilde{\theta}^{2}}\right)$,

directly affected by the PCS parameter $\theta$. Equation (37) also exhibits the same criticality as PQED when we turn the $\theta$ factor off (blue point in Fig. 4) and in the strong-coupling limit, where it also reproduces QED3 for $\theta=0$.

Notice that $N_{c}$ must be at least larger than 1 in order to obtain a broken phase; otherwise, the condition $N<N_{c}$ may not be satisfied. Such a minimum value for $N_{c}$ to permit a symmetry-broken phase $\left(N_{c} \rightarrow 1\right)$ can also be plugged into Eq. (37) to estimate a threshold value in the effective PCS mass $\tilde{\theta}$ given by

$$
\tilde{\theta}_{t h}=\Lambda \sqrt{N_{c}^{\mathrm{PQED}}-1}
$$

This threshold value separates the two different phases that might appear in the system: If $\tilde{\theta} \geq \tilde{\theta}_{t h}$, the system forcibly falls upon its symmetric phase, while $\tilde{\theta}<\tilde{\theta}_{t h}$ allows the formation of a broken phase, as we see in Fig. 4.

\section{NUMERICAL ANALYSIS}

Even though $\Sigma(p)$ analytical solutions could only be obtained approximately in the previous sections, the integral in Eq. (15) needs no approximation to be numerically evaluated. Hence, we can apply the same numeric calculation described in Refs. [54,104] to get a glimpse of the features related with the mass generation in the PMCS model, as illustrated in Figs. 5, 7, and 8.

As illustrated in Fig. 5, the mass function generated is maximum for small momentum values and gets strongly

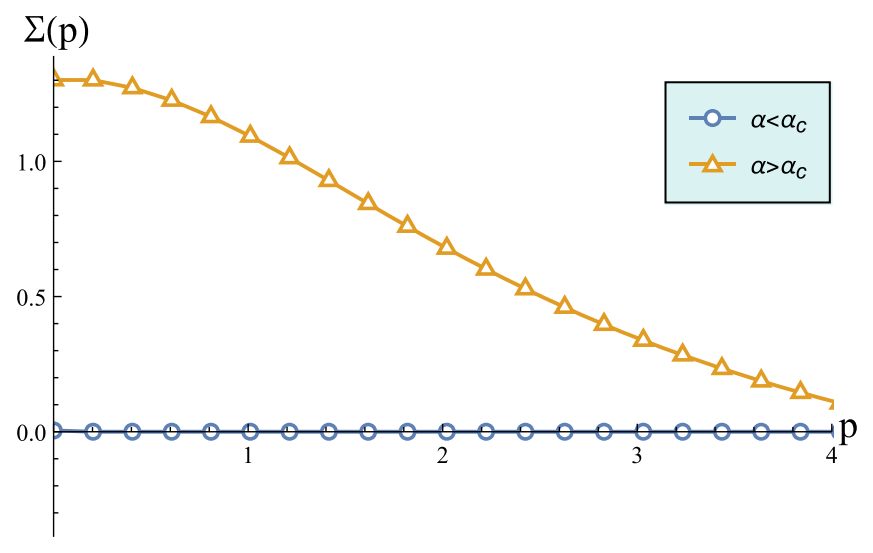

FIG. 5. The mass function generated by the PMCS model above and below its critical point $\alpha_{c}(\theta)$. Plotted is the numerical solution of Eq. (15) with $\theta=0.1, \Lambda=10$, and consequently $\alpha_{c} \approx .39$. The blue line $\left[\alpha=0.2<\alpha_{c}(\theta)\right]$ shows that dynamical mass generation is negligible below the critical fine-structure parameter, while the yellow line exhibits the typical behavior above its critical value $\left(\alpha=0.8>\alpha_{c}\right)$.

suppressed below a different $\alpha_{c}(\theta)$ for each PCS mass, as seen from the sudden increase of $\Sigma(0)$ when each system crosses the critical point in Fig. 6; notice that as $\theta$ grows, the minimum value for mass generation $\alpha_{c}(\theta)$ also increases. These transition points can be used to establish a comparison that confirms the existence of a critical parameter exhibited in Fig. 2. This allow us to identify the numerical value of $\alpha_{c}(\theta)$ with the same order as the analytical result in Fig. 2. The numeric deviation comes from the fact that the analytical solution is obtained after linearizing the differential in Eq. (15), while the numerical results contains all of the nonlinear behavior in Eq. (23), affecting its numerical value, but not changing the existence of the critical point we predict.

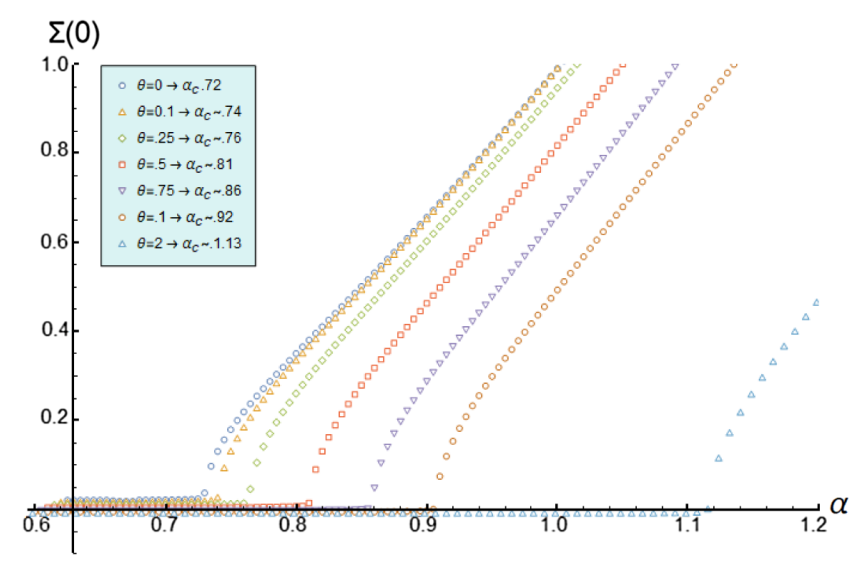

FIG. 6. The small-momentum mass function generated by the PMCS model for different PCS masses. The numerical solution of Eq. (15) is plotted with $\theta$ ranging from 0 to 2 (in units of energy). The estimated values for $\alpha_{c}$ were selected using the values when $\Sigma(0) \approx 0.01$. 


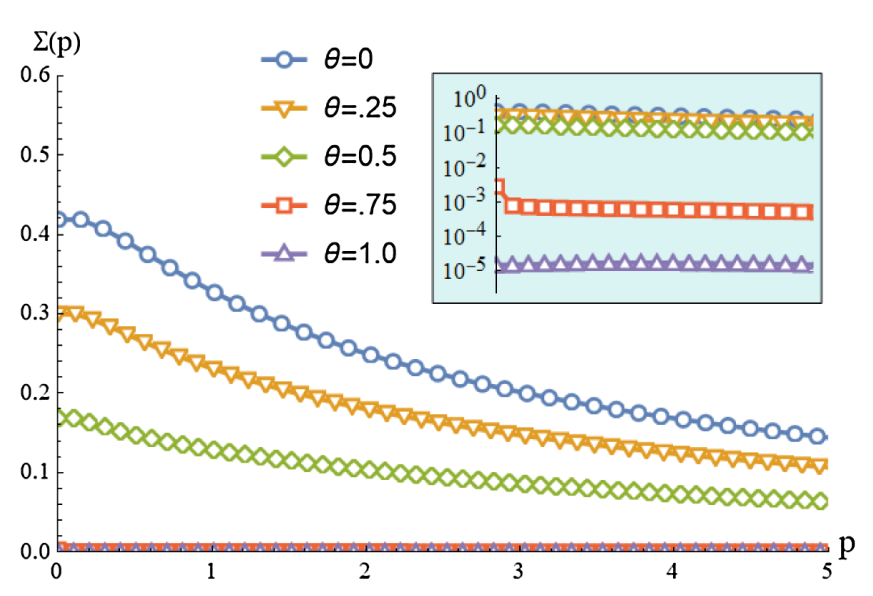

FIG. 7. The mass function generated by the PMCS model for different values of the PCS parameter $\theta$. Plotted is the numerical solution of Eq. (15), assuming $\alpha=0.8>\alpha_{c}(\theta)$ and $\Lambda=10$. The blue inset is the log scale of the same plot to illustrate that the mass generated gets even smaller as $\theta$ approaches 1 .

Considering the system above its critical value $\alpha_{c}$, which allows for mass generation, it is possible to infer that reducing the (inverse-of-mass) interaction range (by means of controlling the PCS mass $\theta$ ) mitigates the mass generation until it becomes almost negligible, as illustrated in Fig. 7.

The qualitative interpretation exhibited in Fig. 4 can be broadened by further analyzing the numerical evaluations of Eq. (37) at different coupling regimes. This illustrates some new features that can be observed in Fig. 8 and clearly sets apart the different behaviors a MPCS system might have. The shaded cyan region is the region where

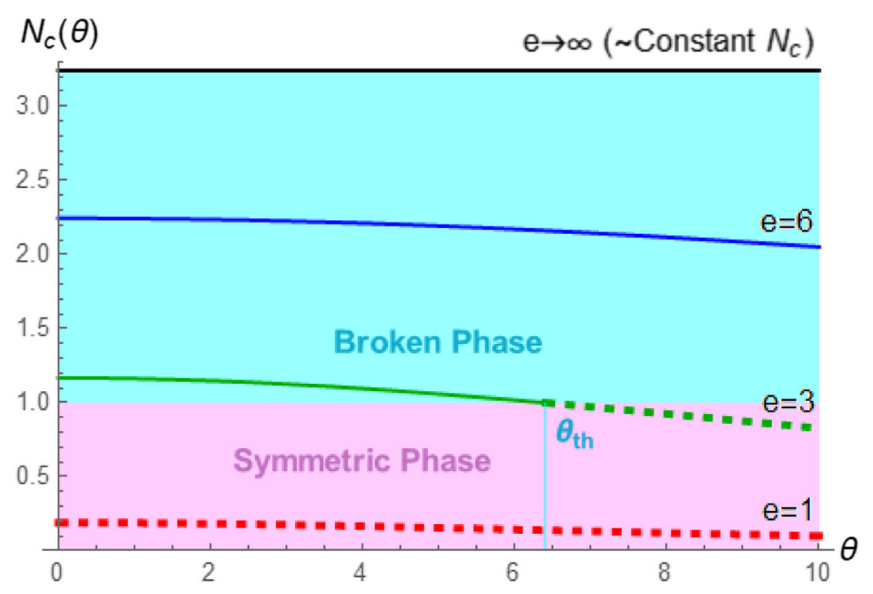

FIG. 8. Dependence of the maximum number of flavors on $\theta$, plotted numerically assuming $\Lambda=10$ and different coupling constants $e$ in Eq. (37). The shaded cyan region $\left[N_{c}(\theta) \geq 1\right]$ marks the potentially breakable states, while the shaded magenta region $\left[N_{c}(\theta) \leq 1\right]$ implies that the system completely falls into its symmetric phase. Notice that the green line $(e=3)$ passes through both regions, which suggests a phase transition around $\theta_{t h}$, as previouly suggested by Fig. 4 .
$N_{c}(\theta)>1$, allowing at least one possible nonzero value for $N$ that breaks the symmetry [i.e., $N_{c}(\theta)>N=1$ ]. Meanwhile, the shaded magenta region $\left[N_{c}(\theta) \leq 1\right]$ implies that the system completely falls into its symmetric phase. As we change the coupling constant $e$, the system critical point travels from a constant $N_{c}(\theta)=N_{C}^{\mathrm{PQED}}$ at its strong-coupling limit (solid black line in Fig. 8) towards an attenuated value that slightly diminishes as $\theta$ increases (solid blue line), until $N_{c}(\theta)$ eventually goes below 1 for all possible $\theta$ (dashed red line). Before it reaches its completely symmetric phase, the system first passes through a peculiar coupling regime with a built-in phase transition (numerically estimated to happen when $2.65<e<3.42$ ). In this regime, the broken-symmetric phase transition appears within a single system, since the broken phase gets forbidden when the mass of the gauge field is above the threshold $\theta_{t h}$ (as indicated by the dashed part of the green line)-i.e., in these coupling regimes, the PCS mass $\theta$ regulates whether the system may or may not have its symmetry broken.

\section{FINAL REMARKS AND PERSPECTIVES}

In this work, we have described the chiral symmetry breaking in the nonperturbative regime of PMCS electrodynamics, a renormalizable model composed by associating PQED with a massive PCS parameter $\theta[98,99]$. We have written the Schwinger-Dyson equations for the model assuming the planar Dirac fermions were four-component spinors and have applied the rainbow-quenched and the unquenched approximations to determine the criticality within the system.

At the rainbow-quenched approximation, we have deduced an analytical expression for the dynamical mass $\Sigma(p)$ [Eq. (24)] and for the critical fine-structure constant $\alpha_{c}$ [Eq. (26)], emphasizing their dependence on $\theta$ to exhibit the correction (proportional to $\theta^{2} / \Lambda^{2}$ ) to $\alpha_{c}^{\mathrm{PQED}}$ caused by the PCS term-whence we deduce that $\alpha_{c}(\theta, \Lambda)$ grows as we enhance the PCS contribution, even though such criticality correction diminishes conversely at larger energy scales, effectively retrieving $\alpha_{c}^{\mathrm{PQED}}$. It is also worth noting that very large $\theta$ values inhibit the dynamical mass generation, since this brings the system to $\alpha<\alpha_{c}$, favoring the symmetric phase.

At the unquenched approximation, we have considered $N$ copies of the Dirac spinors to calculate the critical number of flavors $N_{c}$, governed by Eq. (37). The dependence of the critical parameter on the PCS term in this approximation works the other way around-i.e., $N_{c}$ decreases towards zero as $\theta$ grows for a given $\Lambda$-by setting an upper value for $N$ and $\theta$ [see Eq. (38)]. Meanwhile, the vacuum polarization onto the gauge field propagator not only produces a screening effect, but also modifies the mass of the gauge field according to Eq. (33). In the strong-coupling limit $(e>3.42)$, this mass 
disappears, and then the mass function is the same as in standard PQED in Ref. [54], while weaker couplings either go completely symmetric $(e<2.65)$ or present a model with a phase transition at $\theta=\theta_{\text {th }}(2.65<e<3.42)$. Comparing the $N_{c}$ results for the PMCS electrodynamics with the usual Maxwell-Chern-Simons model (see Appendix A) discloses that they both have the same structure. Differently from the PMCS, however, the Maxwell-Chern-Simons model has a characteristic energy scale (defined by its fine-structure constant), which reflects the fact that this model is super renormalizable.

Both approximations reproduce PQED in the continuum limit, since $\theta \ll \Lambda \rightarrow \infty$. These recursive results regarding PQED are also obtained once we turn the PCS mass off $(\theta \rightarrow 0)$, retrieving $\alpha_{c} \rightarrow \alpha_{c}^{\mathrm{PQED}}$ and $N_{c} \rightarrow N_{c}^{\mathrm{PQED}}$. Thence, we hope that the PMCS model described here will inherit the utility of PQED for the characterization of different kinds of two-dimensional materials, but now with the extra feature of applying to systems with short-range interactions, because $\theta$ reduces the strength of the static potential, possibly including superconductivity and bound states [98], as happens in magic-angle twisted bilayer graphene $[105,106]$ and hybrid graphene/LiNbO3 platforms [107].

\section{ACKNOWLEDGMENTS}

R.F. O. and G.C.M. are partially supported by Coordenação de Aperfeiçoamento de Pessoal de Nível Superior-Brasil (CAPES), finance code 001; R. F. O., V.S. A., and L. O. N. by CAPES/NUFFIC, finance code 0112; and V.S. A. and L. O. N. by research grants from Conselho Nacional de Desenvolvimento Científico e Tecnológico (CNPq).

\section{APPENDIX A: THE QED WITH A CS TERM}

In this appendix, we discuss the main results regarding the dynamical symmetry breaking of MCS theory [92-94]. Our main idea is to show that the $\bar{\theta}$ parameter also favors the symmetric phase. The action of the model, in the Euclidean space, reads

$$
\begin{aligned}
\mathcal{L}_{\mathrm{MCS}}= & \frac{1}{4} \bar{F}^{\mu \nu} \bar{F}_{\mu \nu}+\frac{\lambda}{2}\left(\partial^{\mu} \bar{A}_{\mu}\right)^{2}-i \frac{\bar{\theta}}{2} \epsilon_{\mu \nu \alpha} \bar{A}^{\mu} \partial^{\nu} \bar{A}^{\alpha} \\
& +\bar{\psi}_{a}\left(i \gamma^{\mu} \partial_{\mu}+\bar{e} \gamma^{\mu} \bar{A}_{\mu}\right) \psi_{a},
\end{aligned}
$$

where $\bar{A}_{\mu}$ is a gauge field (usually called the MaxwellChern-Simons field), $\bar{\theta}$ is the CS parameter with units of mass $[\bar{\theta}]=M, \bar{e}$ is the coupling constant with units given by $[\bar{e}]=M^{1 / 2}, \lambda$ is the gauge-fixing parameter, and $\bar{F}_{\mu \nu}=\partial_{\mu} \bar{A}_{\nu}-\partial_{\nu} \bar{A}_{\mu}$ is the strength-field tensor of $\bar{A}_{\mu}$. The matter field is given by the Dirac field $\psi$, and $\gamma_{\mu}$ are the four-rank Dirac matrices, obeying the same properties as in Secs. II-III. Here, we consider a flavor index $a=1, \ldots, N$, which shows that we have $N$ copies of the matter field.

We shall follow the same steps as we have done in the case of the PMCS model. Hence, let us summarize the main results. Within the large- $N$ expansion, at lowest order, the gauge-field propagator reads

$$
\Delta_{\mu \nu}(p)=\Delta(p)\left(\delta_{\mu \nu}-\frac{p_{\mu} p_{\nu}}{p^{2}}\right)+L(p) \epsilon_{\mu \alpha \nu} p^{\alpha},
$$

where

$$
\Delta(p)=\frac{p^{2}+\frac{\bar{e}^{2}}{8}|p|}{p^{2}\left[\left(|p|+\frac{\bar{e}^{2}}{8}\right)^{2}+\bar{\theta}^{2}\right]}
$$

and

$$
L(p)=\frac{\bar{\theta}}{\left[\left(p^{2}\right)^{2}+2\left(p^{2}\right)^{3 / 2} \frac{\bar{e}^{2}}{8}+p^{2} \frac{\bar{e}^{4}}{64}+p^{2} \bar{\theta}^{2}\right]} .
$$

Using Eqs. (A3) and (A4) in the Schwinger-Dyson equation of the electron propagator, one may conclude, after some work, that the mass function $\Sigma(p)$ obeys an integral equation, given by

$\Sigma(p)=\frac{\bar{e}^{2}}{4 N \pi^{2}} \int_{0}^{\infty} d k \frac{k \Sigma(k)}{k^{2}+\Sigma^{2}(k)} \frac{1}{p} \ln \left[\frac{\left(p+k+\frac{\bar{e}^{2}}{8}\right)^{2}+\bar{\theta}^{2}}{\left(|p-k|+\frac{\bar{e}^{2}}{8}\right)^{2}+\bar{\theta}^{2}}\right]$.

Equation (A5) admits an expansion in its kernel, such that one may convert this integral equation into a differential equation. To achieve that, we expand the logarithm in Eq. (A5), assuming $\bar{e}^{2} \gg p$ (because chiral symmetry breaking usually occurs for strong interactions), to derive the property

$$
\begin{aligned}
& \ln \left[\frac{\left(p+k+\frac{\bar{e}^{2}}{8}\right)^{2}+\bar{\theta}^{2}}{\left(|p-k|+\frac{\bar{e}^{2}}{8}\right)^{2}+\bar{\theta}^{2}}\right] \\
& \approx \frac{32 k \bar{e}^{2} \Theta(p-k)}{64 k^{2}+\bar{e}^{4}+64 \bar{\theta}^{2}}+\frac{32 p\left(8 k+\bar{e}^{2}\right) \Theta(k-p)}{64 k^{2}+\bar{e}^{4}+64 \bar{\theta}^{2}} .
\end{aligned}
$$

This approximation has also been applied for the usual QED3 in Refs. [25-36] and in the QED3 coupled to the usual CS term in Refs. [92-94].

Applying the property in Eq. (A6) to the MCS mass function in Eq. (A5), we get

$$
\begin{aligned}
\Sigma(p)= & \frac{\bar{e}^{2}}{4 \pi^{2} N}\left[\int_{0}^{p} d k \frac{k^{2} \Sigma(k)}{k^{2}+\Sigma^{2}(k)} \frac{1}{p} \frac{32 \bar{e}^{2}}{64 k^{2}+\bar{e}^{4}+64 \bar{\theta}^{2}}\right. \\
& \left.+\int_{p}^{\infty} d k \frac{k \Sigma(k)}{k^{2}+\Sigma^{2}(k)} \frac{32\left(8 k+\bar{e}^{2}\right)}{64 k^{2}+\bar{e}^{4}+64 \bar{\theta}^{2}}\right],
\end{aligned}
$$


which can, in turn, be translated to the second-order differential equation, whose linearized version for $p^{2} \gg \Sigma^{2}(p)$ is given by

$$
\frac{d}{d p}\left[p^{2} \frac{d \Sigma(p)}{d p}\right]+\frac{8 \bar{e}^{4}}{N \pi^{2}} \frac{\Sigma(p)}{\bar{e}^{4}+64 \bar{\theta}^{2}}=0,
$$

supplemented by two asymptotic conditions similar to Eqs. (21) and (22).

With that in mind, we obtain the critical number of fermions $N_{c}$, given by

$N_{c}(z)=N_{c}^{\mathrm{QED} 3}\left(\frac{\bar{e}^{4}}{\bar{e}^{4}+64 \bar{\theta}^{2}}\right)=N_{c}^{\mathrm{QED} 3} \frac{1}{\left(1+z^{2}\right)}$,

where $z^{2}=64 \bar{\theta}^{2} / \bar{e}^{4}$. It is interesting to highlight that within the strong-coupling limit-i.e., $\bar{e}^{2} \gg \bar{\theta}$-we obtain $N_{c}^{\mathrm{QED} 3} \rightarrow 32 / \pi^{2} \approx 3.24$ as in Eq. (37). Thence, solving the equation $N_{c}\left(z_{c}\right)=1$, we obtain $z_{c}=\sqrt{32 / \pi^{2}-1}$, which in terms of $\bar{\theta}$ implies that we must have $\bar{\theta}<\bar{\theta}_{t h}$, where $\bar{\theta}_{t h}=z_{c} \bar{e}^{2} / 8$. Therefore, we conclude that the presence of $\bar{\theta} \neq 0$ decreases $N_{c}$ and favors the symmetric phase. For $\alpha \gg 1$, Eq. (38) reproduces the same $\theta_{t h}$ (after using $\Lambda=\bar{e}^{2} / 8$ ), which is a surprising connection between PMCS and MCS that appears in spite of the differences of their actions.

\section{APPENDIX B: ANALYTICAL APPROXIMATION}

After taking the derivative of Eq. (19) with respect to $p$, we find

$\frac{d \Sigma(p)}{d p}=-\frac{\alpha}{\pi p^{2}} \int_{0}^{p} \frac{2 k^{2} \Sigma(k)}{k^{2}+\Sigma^{2}(k)}\left[1-\frac{\theta^{2}}{k^{2}+\theta^{2}}\right] d k$.

From Eqs. (B1) and (19), it follows that the UV and IR conditions are as given by Eqs. (21) and (22), respectively. Thereafter, we multiply Eq. (B1) by $p^{2}$ and calculate the derivative with respect to $p$ again to obtain Eq. (20). This is a nonlinear differential equation, whose solution $\Sigma(p)$ approximately behaves as the solution of our full integral equation in Eq. (15) when the UV/IR conditions are respected.

Obviously, in order to obtain an analytical solution, we have to linearize the differential equation. When we consider $p \ll \Sigma(p)$ in Eq. (20), we find $\Sigma(p)=C_{1} / p+C_{2}$, where $C_{1}$ and $C_{2}$ are arbitrary constants. However, after using the conditions in Eqs. (21) and (22), we conclude that $C_{1}=C_{2}=0$, implying that such approximation does not provide any nontrivial solution that fulfills the auxiliary conditions, and hence the dynamical mass generation is not expected to occur.
Next, let us consider our main case where $p \gg \Sigma(p)$. In this case, the solution $\Sigma(p)$ is given by Eq. (24). Here, because the behavior of the solutions depend on $\lambda_{ \pm}\left(\alpha, \alpha_{c}\right)$, making the analysis more subtle. Within the continuum limit $\Lambda=\infty$, we find that $\Sigma(p) \neq 0$ regardless of the value of $\alpha$, because both the UV and IR conditions are respected.

When $\Lambda$ is finite, it is useful to write the solution as

$$
\Sigma(p)=A p^{(-1+\gamma) / 2}+B p^{(-1-\gamma) / 2},
$$

where $(-1+\gamma) / 2=\lambda_{+}, \quad(-1-\gamma) / 2=\lambda_{-}, \quad$ and $\gamma=$ $\sqrt{1-\alpha / \alpha_{c}}$. Equation (B2) respects the IR condition for any value of $\gamma$ (and therefore $\alpha$ ). To discuss the UV condition, we must first define the range of $\alpha$. For $\alpha<\alpha_{c}$, it follows that the UV condition is not respected; hence, $\Sigma(p)=0$, and the system remains in the symmetric phase. Next, let us consider $\alpha>\alpha_{c}$ so that Eq. (B2) becomes

$$
\Sigma(p)=A p^{(-1+i R) / 2}+B p^{(-1-i R) / 2},
$$

where $R=i \gamma=\sqrt{\alpha / \alpha_{c}-1}$ is a real constant. After some algebra, it is possible to write Eq. (B3) as

$$
\Sigma(p)=\frac{\bar{A}}{\sqrt{p}} \sin \left[R\left(\ln \frac{p}{M_{\Lambda}}+\phi\right)\right],
$$

where $\bar{A}=\sqrt{S^{2}-D^{2}}, \quad S=A+B, \quad D=B-A, \quad$ and $R \phi=\tan ^{-1}(-i S / D)$. The parameter $M_{\Lambda}$ is needed in order to write a dimensionless argument of the logarithmic function. This may be fixed by the UV condition, yielding

$$
M_{\Lambda}=\Lambda e^{2+\phi} e^{-2 n \pi / R}
$$

where $n$ is a positive integer. Note that $M_{\Lambda}$ obeys a Miransky scaling law as in QED4 [72-74,108-110]-i.e., in our results, $\alpha_{c}$ might be interpreted as an ultraviolet fixed point defining the continuum limit of the theory. While in the regime $\alpha>\alpha_{c}$, we find a nontrivial solution and the system is in its broken phase.

One important point regarding the interpretation of $\alpha_{c}$ is the validity of the approximation $p^{2} \gg \Sigma^{2}(p)$. In general grounds, the mass function decreases as we increase $p$-i.e., its maximum value is $\Sigma(0)$. As a consequence, it is unclear whether we actually find $\Sigma(0) \rightarrow 0$ at $\alpha=\alpha_{c}$, since our approach seems to work well only at large $p$. It turns out that we could improve our approximation by considering $p^{2}+\Sigma(p)^{2} \approx p^{2}+\Sigma(0)^{2}$, which also works reasonably well at small external momentum. In this case, we would obtain that $\Sigma(0)$ obeys a Miransky scaling law similar to Eq. (B4), with the very same value of $\alpha_{c}$. Therefore, we do not find any better result for $\alpha_{c}$, but we avoid the limitation of large external momentum, as is done in the context of QED3 in Refs. [100,101]. 
[1] S. Girvin and R. Prange, The Quantum Hall Effect (Springer, New York, 1987).

[2] F. Wilczek, Fractional Statistics and Anyon Superconductivity (World Scientific, Singapore, 1990), Vol. 5.

[3] B. I. Halperin, Phys. Rev. B 25, 2185 (1982).

[4] R. B. Laughlin, Phys. Rev. B 23, 5632 (1981).

[5] R. Laughlin, in Ten Years of Superconductivity: 19801990 (Springer, New York, 1988), pp. 313-316.

[6] Y.-H. Chen, F. Wilczek, E. Witten, and B. I. Halperin, Int. J. Mod. Phys. B 03, 1001 (1989).

[7] K. Farakos and N. E. Mavromatos, Mod. Phys. Lett. A 13, 1019 (1998).

[8] I. F. Herbut, Phys. Rev. B 66, 094504 (2002).

[9] M. Franz, Z. Tešanović, and O. Vafek, Phys. Rev. B 66, 054535 (2002).

[10] D. J. Thouless, M. Kohmoto, M. P. Nightingale, and M. Den Nijs, Phys. Rev. Lett. 49, 405 (1982).

[11] F. D. M. Haldane, Phys. Rev. Lett. 61, 2015 (1988).

[12] P. A. Lee, N. Nagaosa, and X.-G. Wen, Rev. Mod. Phys. 78, 17 (2006).

[13] I. Affleck and J. B. Marston, Phys. Rev. B 37, 3774 (1988).

[14] L. B. Ioffe and A. I. Larkin, Phys. Rev. B 39, 8988 (1989).

[15] D. H. Kim, P. A. Lee, and X.-G. Wen, Phys. Rev. Lett. 79, 2109 (1997).

[16] D. H. Kim and P. A. Lee, Ann. Phys. (N.Y.) 272, 130 (1999).

[17] W. Rantner and X.-G. Wen, Phys. Rev. Lett. 86, 3871 (2001).

[18] M. Franz and Z. Tešanović, Phys. Rev. Lett. 87, 257003 (2001).

[19] I. F. Herbut, Phys. Rev. Lett. 88, 047006 (2002).

[20] G.-Z. Liu and G. Cheng, Phys. Rev. B 66, 100505(R) (2002).

[21] G.-Z. Liu and G. Cheng, Phys. Rev. D 67, 065010 (2003).

[22] B. A. Bernevig and T. L. Hughes, Topological Insulators and Topological Superconductors (Princeton University Press, Princeton, NJ, 2013).

[23] S.-Q. SHEN, Topological Insulators: Dirac Equation in Condensed Matter (Springer, New York, 2018).

[24] M. Z. Hasan and C. L. Kane, Rev. Mod. Phys. 82, 3045 (2010).

[25] R. D. Pisarski, Phys. Rev. D 29, 2423 (1984).

[26] T. Appelquist, M. J. Bowick, D. Karabali, and L. Wijewardhana, Phys. Rev. D 33, 3774 (1986).

[27] T. Appelquist, D. Nash, and L. C. R. Wijewardhana, Phys. Rev. Lett. 60, 2575 (1988).

[28] D. Nash, Phys. Rev. Lett. 62, 3024 (1989).

[29] Y. Hoshino and T. Matsuyama, Phys. Lett. B 222, 493 (1989).

[30] E. Dagotto, J. B. Kogut, and A. Kocić, Phys. Rev. Lett. 62 , 1083 (1989).

[31] E. Dagotto, A. Kocić, and J. B. Kogut, Nucl. Phys. B334, 279 (1990).

[32] M. R. Pennington and D. Walsh, Phys. Lett. B 253, 246 (1991).

[33] D. C. Curtis, M. R. Pennington, and D. Walsh, Phys. Lett. B 295, 313 (1992).

[34] D. Atkinson, P. W. Johnson, and P. Maris, Phys. Rev. D 42, 602 (1990).
[35] K.-i. Kondo and H. Nakatani, Prog. Theor. Phys. 87, 193 (1992).

[36] C. J. Burden and C. D. Roberts, Phys. Rev. D 44, 540 (1991).

[37] C. J. Burden, J. Praschifka, and C. D. Roberts, Phys. Rev. D 46, 2695 (1992).

[38] G. Grignani, G. Semenoff, and P. Sodano, Phys. Rev. D 53, 7157 (1996).

[39] P. Maris, Phys. Rev. D 52, 6087 (1995).

[40] E. C. Marino, L. O. Nascimento, V.S. Alves, and C. Morais Smith, Phys. Rev. D 90, 105003 (2014).

[41] R. Do Amaral and E. C. Marino, J. Phys. A 25, 5183 (1992).

[42] D. G. Barci, L. E. Oxman, and M. Rocca, Int. J. Mod. Phys. A 11, 2111 (1996).

[43] K. S. Novoselov, A. K. Geim, S. V. Morozov, D. Jiang, Y. Zhang, S. V. Dubonos, I. V. Grigorieva, and A. A. Firsov, Science 306, 666 (2004).

[44] K. S. Novoselov, D. Jiang, F. Schedin, T. Booth, V. Khotkevich, S. Morozov, and A. K. Geim, Proc. Natl. Acad. Sci. U.S.A. 102, 10451 (2005).

[45] A. K. Geim and K. S. Novoselov, in Nanoscience and Technology: A Collection of Reviews from Nature Journals (World Scientific, Singapore, 2010), pp. 11-19.

[46] A. H. Castro Neto, F. Guinea, N. M. R. Peres, K. S. Novoselov, and A. K. Geim, Rev. Mod. Phys. 81, 109 (2009).

[47] F. Juan, A. G. Grushin, and M. A. H. Vozmediano, Phys. Rev. B 82, 125409 (2010).

[48] M. A. H. Vozmediano and F. Guinea, Phys. Scr. T146, 014015 (2012).

[49] B. Lalmi, H. Oughaddou, H. Enriquez, A. Kara, S. Vizzini, B. Ealet, and B. Aufray, Appl. Phys. Lett. 97, 223109 (2010).

[50] G. Wang, A. Chernikov, M. M. Glazov, T. F. Heinz, X. Marie, T. Amand, and B. Urbaszek, Rev. Mod. Phys. 90, 021001 (2018).

[51] L. Li, Y. Yu, G. J. Ye, Q. Ge, X. Ou, H. Wu, D. Feng, X. H. Chen, and Y. Zhang, Nat. Nanotechnol. 9, 372 (2014).

[52] X.-S. Ye, Z.-G. Shao, H. Zhao, L. Yang, and C.-L. Wang, RSC Adv. 4, 21216 (2014).

[53] Q. H. Wang, K. Kalantar-Zadeh, A. Kis, J. N. Coleman, and M. S. Strano, Nat. Nanotechnol. 7, 699 (2012).

[54] V. S. Alves, W. S. Elias, L. O. Nascimento, V. Juričić, and F. Peña, Phys. Rev. D 87, 125002 (2013).

[55] L. O. Nascimento, V. S. Alves, F. Peña, C. M. Smith, and E. C. Marino, Phys. Rev. D 92, 025018 (2015).

[56] V.S. Alves, R. O. C. Junior, E. C. Marino, and L. O. Nascimento, Phys. Rev. D 96, 034005 (2017).

[57] E. C. Marino, L. O. Nascimento, V.S. Alves, and C. M. Smith, Phys. Rev. X 5, 011040 (2015).

[58] N. Menezes, V. S. Alves, E. C. Marino, L. Nascimento, L. O. Nascimento, and C. M. Smith, Phys. Rev. B 95, 245138 (2017).

[59] E. C. Marino, L. O. Nascimento, V. S. Alves, N. Menezes, and C. M. Smith, 2D Mater. 5, 041006 (2018).

[60] A. Chaves, R. Ribeiro, T. Frederico, and N. Peres, 2D Mater. 4, 025086 (2017). 
[61] S. Teber and A. V. Kotikov, Theor. Math. Phys. 190, 446 (2017).

[62] L. Fernández, R. O. Corrêa Jr, V. S. Alves, L. O. Nascimento, and F. Peña, Phys. Rev. D 103, 025018 (2021).

[63] V.S. Alves, T. Macri, G. C. Magalhães, E. Marino, and L. O. Nascimento, Phys. Rev. D 97, 096003 (2018).

[64] R. Ozela, V.S. Alves, E. Marino, L. O. Nascimento, J. Neto, R. O. Ramos, and C. M. Smith, arXiv:1907.11339.

[65] J. D. L. Silva, A. N. Braga, W. P. Pires, V. S. Alves, D. T. Alves, and E. Marino, Nucl. Phys. B920, 221 (2017).

[66] W. P. Pires, J. D. L. Silva, A. N. Braga, V. S. Alves, D. T. Alves, and E. Marino, Nucl. Phys. B932, 529 (2018).

[67] K. Johnson, M. Baker, and R. Willey, Phys. Rev. 136, B1111 (1964).

[68] T. Maskawa and H. Nakajima, Prog. Theor. Phys. 52, 1326 (1974).

[69] R. Fukuda and T. Kugo, Nucl. Phys. B117, 250 (1976).

[70] D. Curtis and M. R. Pennington, Phys. Rev. D 42, 4165 (1990).

[71] D. Atkinson, V. Gusynin, and P. Maris, Phys. Lett. B 303, 157 (1993).

[72] P. Fomin, V. Gusynin, V. Miransky, and Y. A. Sitenko, Riv. Nuovo Cimento (1978-1999) 6, 1 (1983).

[73] V. Miransky, Phys. Lett. 165B, 401 (1985).

[74] V. Miransky, Il Nuovo Cimento A (1965-1970) 90, 149 (1985).

[75] J. Kogut, E. Dagotto, and A. Kocic, Phys. Rev. Lett. 60, 772 (1988).

[76] J. B. Kogut, E. Dagotto, and A. Kocić, Nucl. Phys. B317, 253 (1989).

[77] E. Dagotto, J. B. Kogut, and A. Kocić, Phys. Rev. D 43, R1763 (1991).

[78] K.-i. Kondo, Y. Kikukawa, and H. Mino, Phys. Lett. B 220, 270 (1989).

[79] V. Gusynin, Mod. Phys. Lett. A 05, 133 (1990).

[80] A. Bashir, C. Calcaneo-Roldan, L. X. Gutiérrez-Guerrero, and M.E. Tejeda-Yeomans, Phys. Rev. D 83, 033003 (2011).

[81] A. Kızılersü and M. R. Pennington, Phys. Rev. D 79, 125020 (2009).

[82] F. J. Dyson, Phys. Rev. 75, 1736 (1949).

[83] J. Schwinger, Proc. Natl. Acad. Sci. U.S.A. 37, 452 (1951).

[84] C. D. Roberts and A. G. Williams, Prog. Part. Nucl. Phys. 33, 477 (1994).

[85] G. V. Dunne, in Aspects Topologiques de la Physique en Basse Dimension (Springer, New York, 1999), pp. 177-263.
[86] M. Carena, T. Clark, and C. Wagner, Int. J. Mod. Phys. A 06, 217 (1991).

[87] E. Fradkin, Phys. Rev. Lett. 63, 322 (1989).

[88] J. D. Lykken, J. Sonnenschein, and N. Weiss, Phys. Rev. D 42, 2161 (1990).

[89] S. Deser, R. Jackiw, and S. Templeton, Phys. Rev. Lett. 48, 975 (1982).

[90] W. Siegel, Nucl. Phys. B156, 135 (1979).

[91] R. Jackiw and S. Templeton, Phys. Rev. D 23, 2291 (1981).

[92] D. K. Hong and S.H. Park, Phys. Rev. D 47, 3651 (1993).

[93] C. R. A. Williams, Part. Nucl. Phys. 33, 477 (1994).

[94] K.-I. Kondo and P. Maris, Phys. Rev. D 52, 1212 (1995).

[95] G. C. Magalhães, V. S. Alves, E. C. Marino, and L. O. Nascimento, Phys. Rev. D 101, 116005 (2020).

[96] M. E. Carrington, Phys. Rev. B 99, 115432 (2019).

[97] J. A. C. Olivares, L. Albino, A. J. Mizher, and A. Raya, Phys. Rev. D 102, 096023 (2020).

[98] V. S. Alves, E. Marino, L. O. Nascimento, J. M. Neto, R. F. Ozela, and R. O. Ramos, Phys. Lett. B 797, 134860 (2019).

[99] G. C. Magalhães, V. S. Alves, L. O. Nascimento, and E. C. Marino, Phys. Rev. D 103, 116022 (2021).

[100] P. Maris, Phys. Rev. D 54, 4049 (1996).

[101] P. I. Fomin, V. P. Gusynin, and V. A. Miransky, Phys. Lett. 78B, 136 (1978).

[102] R. D. Pisarski, Phys. Rev. D 29, 2423 (1984).

[103] T. W. Appelquist, Prog. Theor. Phys. Suppl. 85, 244 (1985).

[104] L. Fernández, V. S. Alves, L. O. Nascimento, F. Peña, M. Gomes, and E. C. Marino, Phys. Rev. D 102, 016020 (2020).

[105] Z. Song, Z. Wang, W. Shi, G. Li, C. Fang, and B. A. Bernevig, Phys. Rev. Lett. 123, 036401 (2019).

[106] Y. Sheffer and A. Stern, Phys. Rev. B 104, L121405 (2021).

[107] Z. Yu, Y. Wang, B. Sun, Y. Tong, J.-B. Xu, H. K. Tsang, and $\mathrm{X}$. Sun, in CLEO: Science and Innovations (Optical Society of America, Washington, DC, 2020), pp. STh1F-7.

[108] V. A. Miransky and K. Yamawaki, Phys. Rev. D 55, 5051 (1997).

[109] W. A. Bardeen, C. N. Leung, and S. T. Love, Phys. Rev. Lett. 56, 1230 (1986).

[110] V. A. Miransky and K. Yamawaki, Mod. Phys. Lett. A 04, 129 (1989). 